\title{
Phase formation in intermixed Ni-Ge thin films: Influence of Ge content and low-temperature nucleation of hexagonal nickel germanides
}

\author{
B. De Schutter ${ }^{\mathrm{a}, *}$, W. Devulder ${ }^{\mathrm{a}}$, A. Schrauwen ${ }^{\mathrm{b}}$, K. van Stiphout ${ }^{\mathrm{b}}$, T. Perkisas ${ }^{\mathrm{c}}$, S. Bals ${ }^{\mathrm{c}}$, A. Vantomme ${ }^{\mathrm{b}}$, \\ C. Detavernier ${ }^{a}$
}

a Dept. of Solid-State Sciences, Ghent University, Krijgslaan 281/S1, 9000 Ghent, Belgium

${ }^{\mathrm{b}}$ Instituut voor Kern- en Stralingsfysica, KU Leuven, Celestijnenlaan 200D, B-3001 Leuven, Belgium

' EMAT, University of Antwerp, Groenenborgerlaan 171, 2020 Antwerp, Belgium

\section{A R T I C L E I N F O}

\section{Article history:}

Available online 20 September 2013

\section{Keywords:}

Nickel

Germanium

Thin film

Germanides

In situ XRD

Phase formation

\begin{abstract}
A B S T R A C T
In this study, we focus on phase formation in intermixed Ni-Ge thin films as they represent a simplified model of the small intermixed interface layer that is believed to form upon deposition of $\mathrm{Ni}$ on Ge and where initial phase formation happens. A combinatorial sputter deposition technique was used to co-deposit a range of intermixed $\mathrm{Ni}-\mathrm{Ge}$ thin films with $\mathrm{Ge}$ concentrations varying between 0 and 50 at.\%Ge in a single deposition on both $\mathrm{Ge}(100)$ and inert $\mathrm{SiO}_{2}$ substrates. In situ X-ray diffraction and transmission electron microscopy where used to study phase formation. In almost the entire composition range under investigation, crystalline phases where found to be present in the as-deposited films. Between 36 and 48 at.\%Ge, high-temperature hexagonal nickel germanides were found to occur metastabily below $300^{\circ} \mathrm{C}$, both on $\mathrm{SiO}_{2}$ and $\mathrm{Ge}(100)$ substrates. For Ge concentrations in the range between 36 and 42 at.\%, this hexagonal germanide phase was even found to be present at room temperature in the asdeposited films. The results obtained in this work could provide more insight in the phase sequence of a pure Ni film on Ge.
\end{abstract}

(c) 2013 Elsevier B.V. All rights reserved.

\section{Introduction}

The continuous scaling down of microelectronic devices pushes the current Si-based technology to its limits. Alternative materials are being investigated to replace $\mathrm{Si}$ in e.g. the gate regions of MetalOxide-Semiconducter Field Effect Transistors (MOSFET). According to the industry roadmap, $\mathrm{Ge}$ is a top candidate to replace $\mathrm{Si}$ in p-channel MOSFET devices due to its higher carrier mobility and relative compatibility with silicon processing [1]. Similar to the current SALICIDE (Self-Aligned Silicide) process, where metal silicides are used to contact the source, gate and drain regions of MOSFETs, metal germanides appear as natural candidates for contacting Ge. A systematic study of germanide formation and properties in 20 transition metal on Ge systems by Gaudet et al. [2], revealed $\mathrm{NiGe}$ as the most promising contact material since it exhibits the most suitable properties among all investigated metal germanides, including low formation temperature, low resistivity and a wide stable temperature window during ramp anneals.

If we are to use NiGe as a contact material in future devices, a thorough understanding of the formation mechanisms and properties of the different germanides in the $\mathrm{Ni}-\mathrm{Ge}$ system is important.

\footnotetext{
* Corresponding author. Tel.: +32 92644370 .

E-mail address: bob.deschutter@ugent.be (B. De Schutter).
}

For the bulk system, most of the work on this binary Ni-Ge system was done in the 1970s [3,4] and was summarized in 1987 by Nash and Nash [5], which led to the Ni-Ge binary phase diagram as it is known today (see Fig. 1). For the thin film system, detailed studies on the phase formation upon annealing of a thin $\mathrm{Ni}$ film on $\mathrm{Ge}$ using in situ X-ray diffraction (XRD) were performed by Gaudet et al. [6] and Nemouchi et al. [7]. Both groups reported the simultaneous growth of $\mathrm{NiGe}$ and $\mathrm{Ni}_{5} \mathrm{Ge}_{3}$ on $\mathrm{Ge}(100)$, where $\mathrm{Ni}_{5} \mathrm{Ge}_{3}$ only exists over a small temperature window since it is consumed by the growth of NiGe, which is the stable end phase. However, the existence of other Ni-germanides in this intermediate region could not be ruled out due to overlapping peak positions of different $\mathrm{Ni}$ rich germanides [6]. Interestingly, Jensen et al. [8] reported on the metastable nucleation of the high temperature $\epsilon-\mathrm{Ni}_{5} \mathrm{Ge}_{3}$ when annealing ratio-controlled $\mathrm{Ni}-\mathrm{Ge}$ multilayers on an inert $\mathrm{SiO}_{2}$ substrate.

In order to fully understand the phase formation in the $\mathrm{Ni}-\mathrm{Ge}$ system, more information on this Ni-rich phase region is required. In the $\mathrm{Ni}-\mathrm{Si}$ system, a similar region of transient $\mathrm{Ni}$-rich phases during a ramp anneal of a thin Ni film on $\mathrm{Si}$ can be observed. Recently, a detailed study of a ratio-controlled $\mathrm{Ni}-\mathrm{Si}$ system was performed in our group to gain more insight in the early phase formation in the $\mathrm{Ni}-\mathrm{Si}$ system, which led to the identification of the metastable hexagonal $\theta$-phase in the $\mathrm{Ni}$ on $\mathrm{Si}$ phase sequence 


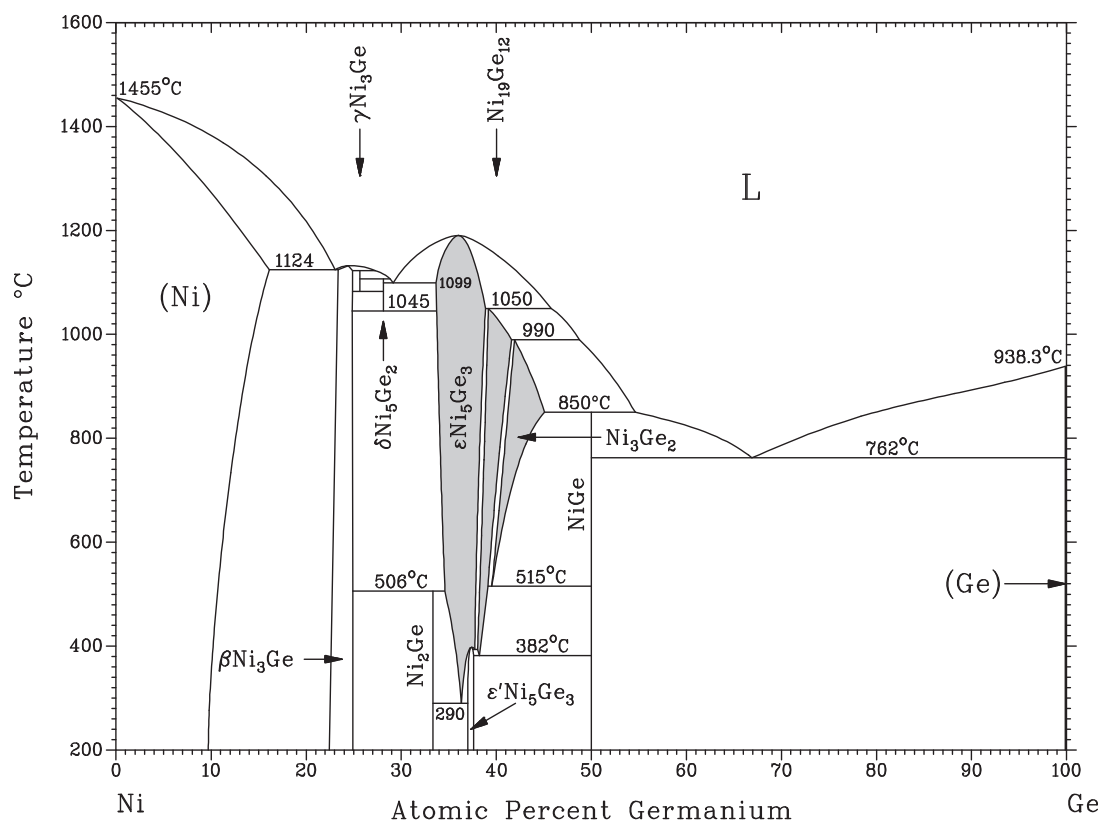

Fig. 1. Equilibrium phase diagram of the Ni-Ge system. This study focuses on the $0-50$ at.\%Ge composition region. (taken from [5]).

[9-12]. In this paper, we present a similar study of phase formation in the $\mathrm{Ni}-\mathrm{Ge}$ system. Ratio-controlled Ni-Ge films were deposited on both $\mathrm{SiO}_{2}$ and $\mathrm{Ge}(100)$ substrates and in situ XRD was used to monitor phase formation during linear ramp anneals.

\section{Experimental}

Intermixed $\mathrm{Ni}-\mathrm{Ge}$ films with varying Ge concentrations (from 0 to 50 at.\%Ge) were deposited on both $100 \mathrm{~nm}$ thermal $\mathrm{SiO}_{2}$ and HFcleaned p-type Ge (100) substrates using a combinatorial sputter deposition technique. Substrate strips with a length of $15 \mathrm{~cm}$ and a width of $1-2 \mathrm{~cm}$ were mounted on a rotating carousel in a deposition chamber with a base pressure of $2 \times 10^{-7}$ mbar. During cosputtering of $\mathrm{Ni}$ and $\mathrm{Ge}$, spatial control of the separate material fluxes resulted in a Ge film with a thickness gradient being intermixed with a uniform Ni film. The deposition parameters were tuned in such a way that the $\mathrm{Ni}$ content in the resulting film is constant and corresponds to the amount of $\mathrm{Ni}$ in a pure $50 \mathrm{~nm}$ Ni film (i.e. about $45 \times 10^{16}$ atoms $/ \mathrm{cm}^{2}$ ). The outcome of such a single deposition is a $15 \mathrm{~cm}$ long strip with a $\mathrm{Ni}-\mathrm{Ge}$ film in which the Ge concentration varies from 0 to 50 at.\%. This gradient is then cleaved into individual $5 \mathrm{~mm}$ long samples for further characterization. This effectively results in 29 samples per deposited strip with a composition difference of $\sim 2$ at.\% between consecutive samples and a Ge concentration uniformity of \pm 1 at.\% within a single sample. The composition of all samples was verified with Rutherford Backscattering Spectrometry (RBS).

Germanide phase formation was studied using a home-built in situ XRD setup, consisting of an experimental heating chamber mounted in a Bruker D8 Discover XRD system. All individual samples were subjected to a ramp anneal at $3{ }^{\circ} \mathrm{C} / \mathrm{s}$ from room temperature up to a temperature of $800^{\circ} \mathrm{C}$ or $650{ }^{\circ} \mathrm{C}$ for the samples on $\mathrm{SiO}_{2}$ and $\mathrm{Ge}(100)$ respectively. During the anneal, the diffraction pattern was recorded every $3 \mathrm{~s}$ over a range of $20^{\circ}$ in $2 \theta$.

High Resolution Transmission Electron Microscopy (HR-TEM) was performed on a FEI Tecnai G2 electron microscope, operating at $200 \mathrm{kV}$. Sample preparation was done using mechanical polishing followed by ion milling.

\section{Results}

\section{1. $\mathrm{Ni}(\mathrm{Ge})$ on inert $\mathrm{SiO}_{2}$ substrates}

Due to the inert $\mathrm{SiO}_{2}$ substrate, the $\mathrm{Ni}-\mathrm{Ge}$ layers have a fixed $\mathrm{Ni} / \mathrm{Ge}$ composition throughout the anneal. As can be expected from the phase diagram (Fig. 1), the amount of $\mathrm{Ge}$ in the $\mathrm{Ni}(\mathrm{Ge})$ mixture will have an influence on the initial crystallization temperature and the first forming phase. Here, in situ XRD was used to probe the phase formation sequence while heating the samples to $800{ }^{\circ} \mathrm{C}$.

Fig. 2(a) shows selected in situ XRD measurements with $2 \theta$ on the vertical axis and temperature on the horizontal axis. The measured XRD intensity is plotted as a logarithmic grayscale map (with black corresponding to the highest intensity). To illustrate the procedure of identifying the phase formation sequence, we will discuss the second in situ XRD scan, i.e. the scan of the sample with 33 at.\%Ge. From room temperature on, three peaks are clearly visible, indicating the presence of a crystalline phase in the as-deposited film. All these peaks can be attributed to $\mathrm{Ni}_{2} \mathrm{Ge}$ : the peak at $2 \theta=41.3^{\circ}$ can be indexed as $\mathrm{Ni}_{2} \mathrm{Ge}(103)$, the intense peak around $2 \theta=44^{\circ}$ is a superposition of $\mathrm{Ni}_{2} \mathrm{Ge}(031)$ and $\mathrm{Ni}_{2} \mathrm{Ge}(211)$ and the third peak around $2 \theta=47.5^{\circ}$ can be indexed as a superposition of $\mathrm{Ni}_{2} \mathrm{Ge}(020)$ and $\mathrm{Ni}_{2} \mathrm{Ge}(113)$. Around $550{ }^{\circ} \mathrm{C}$, a phase transformation occurs as indicated by the disappearance of the three $\mathrm{Ni}_{2} \mathrm{Ge}$ peaks and the emergence of three new peaks. The new features at $2 \theta=43.8^{\circ}$ and $51^{\circ}$ can be identified as $\beta-\mathrm{Ni}_{3} \mathrm{Ge}(111)$ and $\beta-\mathrm{Ni}_{3-}$ $\mathrm{Ge}(200)$ respectively. The third peak around $2 \theta=45^{\circ}$ however is not that straightforward to identify, since it can be indexed as either $\epsilon-\mathrm{Ni}_{5} \mathrm{Ge}_{3}(102), \mathrm{Ni}_{3} \mathrm{Ge}_{2}(102)$ or $\mathrm{Ni}_{19} \mathrm{Ge}_{12}(212)$. A complementary in situ XRD scan (not shown here) in a different $2 \theta$ window revealed a second peak around $2 \theta=30.5^{\circ}$ which can similarly be attributed to the (101) peak of either $\epsilon-\mathrm{Ni}_{5} \mathrm{Ge}_{3}$ or $\mathrm{Ni}_{3} \mathrm{Ge}_{2}$ or to $\mathrm{Ni}_{19}$ $\mathrm{Ge}_{12}(200)$. Similar identification difficulties of these specific phases, which exist over a broad composition range in the binary $\mathrm{Ni}-\mathrm{Ge}$ phase diagram (refer to the shaded area in Fig. 1), were encountered in a vast subset of the measured samples. This identification problem can be related to the very closely related crystal structures of these three phases $[3,13]$, which makes it nearly impossible to discern between them based solely on powder XRD 

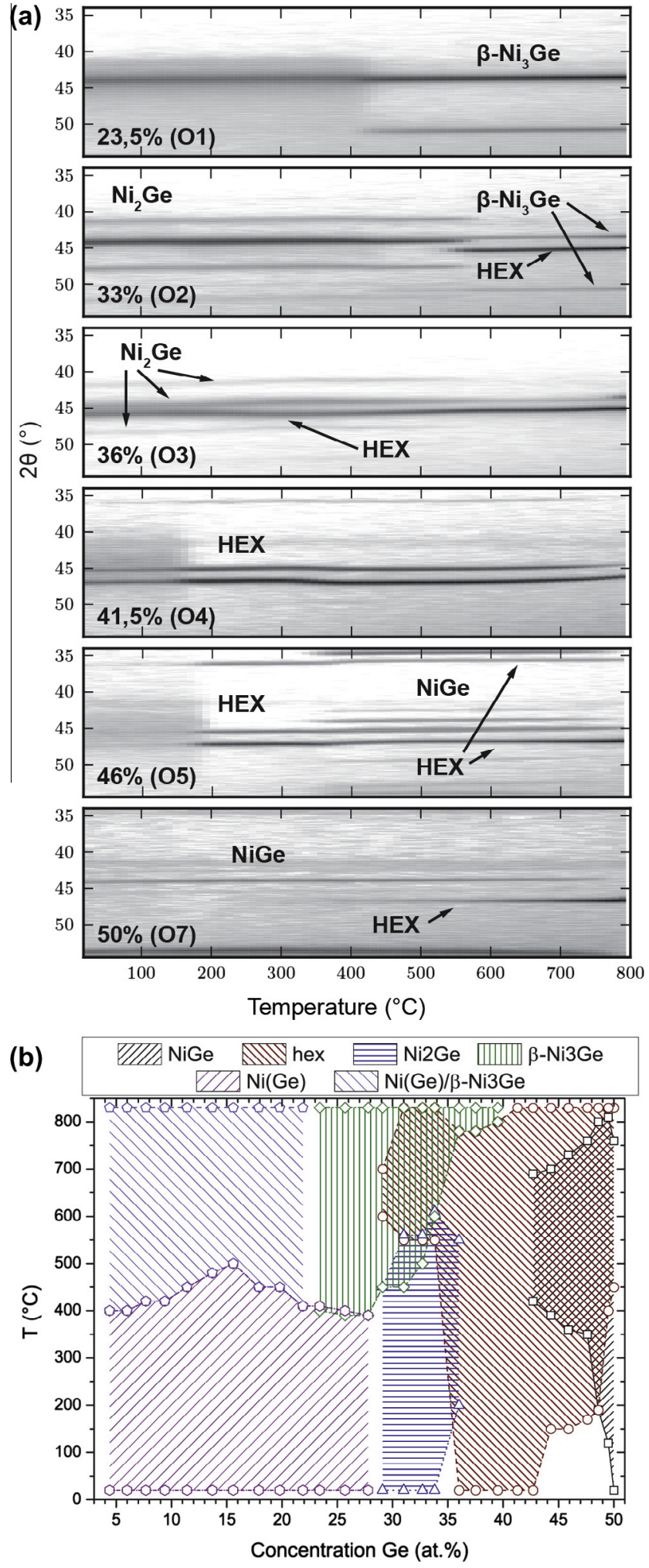

Fig. 2. (a) Selected in situ XRD scans representing the different phase formation sequences observed on the oxide samples during an anneal to $800^{\circ} \mathrm{C}$ at $3^{\circ} \mathrm{C} / \mathrm{s}$. The XRD intensity is plotted as a function of temperature ( $x$-axis) and $2 \theta$ ( $y$-axis) using a grayscale map with black representing the highest intensity. (b) Summary of phase formation for $\mathrm{Ni}(\mathrm{X} \% \mathrm{Ge})$ on $\mathrm{SiO}_{2}$ samples. The shaded areas mark the regions in Ge concentration and temperature where the respective phase was detected with in situ XRD.

techniques. Therefore, in our XRD results, we will depict these phases as one single phase labeled 'HEX', referring to their hexagonal crystal structure.
A similar analysis was performed for all samples, i.e. the phases were identified on the in situ XRD scans and the temperature where a particular phase appears/disappears was systematically recorded. In total, we could discern between seven different phase formation sequences for the samples on $\mathrm{SiO}_{2}$ :

$$
\begin{aligned}
& \text { O1. } \mathrm{Ni}(4-28 \% \mathrm{Ge}) \rightarrow \beta-\mathrm{Ni}_{3} \mathrm{Ge} / \mathrm{Ni}(\mathrm{Ge}) \\
& \text { O2. } \mathrm{Ni}(29-34 \% \mathrm{Ge}) \rightarrow \mathrm{Ni}_{2} \mathrm{Ge} \rightarrow \mathrm{HEX}+\beta-\mathrm{Ni}_{3} \mathrm{Ge} \\
& \text { O3. } \mathrm{Ni}(36 \% \mathrm{Ge}) \rightarrow \mathrm{HEX} \rightarrow \mathrm{Ni}_{2} \mathrm{Ge}+\mathrm{HEX} \rightarrow \mathrm{HEX} \\
& \text { O4. } \mathrm{Ni}(37.5-41.5 \% \mathrm{Ge}) \rightarrow \mathrm{HEX} \\
& \text { O5. } \mathrm{Ni}(42.5-47.5 \% \mathrm{Ge}) \rightarrow \mathrm{HEX} \rightarrow \mathrm{HEX}+\mathrm{NiGe} \rightarrow \mathrm{HEX} \\
& \text { O6. } \mathrm{Ni}(48.5 \% \mathrm{Ge}) \rightarrow \mathrm{HEX}+\mathrm{NiGe} \\
& \text { O7. } \mathrm{Ni}(49-50 \% \mathrm{Ge}) \rightarrow \mathrm{NiGe} \rightarrow \mathrm{NiGe}+\mathrm{HEX}
\end{aligned}
$$

Representative in situ XRD measurements for these cases are shown in Fig. 2(a) (a measurement representing case 06 has been left out since it is the same as for case 07, apart from the as-deposited NiGe phase). For most compositions, the phase sequence can be understood by looking at the relevant region in the Ni-Ge equilibrium phase diagram. However, for samples with a Ge concentration between 36 and 48 at.\% (cases 03 through 06), the HEX phase which should only be stable above $300^{\circ} \mathrm{C}$ is surprisingly observed to crystallize below $300{ }^{\circ} \mathrm{C}$. For samples with a Ge concentration between 36 at.\% and 43 at.\%, the HEX phase is even present asdeposited (see Fig. 2), indicating that this phase was formed during co-deposition of $\mathrm{Ni}$ and $\mathrm{Ge}$ at room temperature. Since one could wonder whether the room temperature HEX phase formation is related to the deposition technique used to co-deposit the films, we investigated $\mathrm{Ni}-\mathrm{Ge}$ films with Ge concentrations of 40 and 42 at.\% that were co-deposited on $\mathrm{SiO}_{2}$ using Molecular Beam Epitaxy. Also in this case, XRD results (not shown here) clearly revealed the presence of a crystalline HEX phase in the as-deposited state.

In Fig. 2(b), a complete phase formation summary for the samples on $\mathrm{SiO}_{2}$ is presented. For each identified phase, the respective shaded areas give the region in both temperature and Ge concentration where this particular phase was visible on the in situ XRD scans. Within the white regions on this plot, no diffraction peaks were observed, indicating an amorphous mixture.

\section{2. $\mathrm{Ni}(\mathrm{Ge})$ on reactive $\mathrm{Ge}(100)$ substrates}

On Ge (100), the phase formation sequence will be influenced by the unlimited supply of Ge from the substrate. We expect the initial crystallization of the film to be similar to the corresponding sample deposited on $\mathrm{SiO}_{2}$. Subsequently, the reaction proceeds by consuming $\mathrm{Ge}$ from the substrate until the whole film has transformed to NiGe, which is expected to be the end phase according to the binary phase diagram.

A similar analysis as for the samples on $\mathrm{SiO}_{2}$ was performed for the samples on Ge (100). Fig. 3(b) shows a phase formation summary for these samples. Below 28 at.\%Ge, the observed phase sequence is qualitatively the same as what has been observed previously for the reaction of a pure Ni film on Ge (100) [6]. Adding over 28 at.\%Ge results in a phase sequence with successive phases increasing in $\mathrm{Ge}$ content. As for the samples on $\mathrm{SiO}_{2}$, the phase sequence systematically changes when more $\mathrm{Ge}$ is added to the as-deposited film. In summary, we observed the following phase formation sequences for the samples on Ge (100):

G1. Ni (4-28\%Ge) $\rightarrow$ Ni-rich phase $(\mathrm{s}) \rightarrow \mathrm{NiGe}$

G2. $\mathrm{Ni}(29-34 \% \mathrm{Ge}) \rightarrow \mathrm{Ni}_{2} \mathrm{Ge} \rightarrow \mathrm{HEX}+\mathrm{NiGe} \rightarrow \mathrm{NiGe}$

G3. Ni $(36 \% \mathrm{Ge}) \rightarrow \mathrm{Ni} 2 \mathrm{Ge}+\mathrm{HEX} \rightarrow \mathrm{HEX}+\mathrm{NiGe} \rightarrow \mathrm{NiGe}$

G4. Ni (37.5-47.5\%Ge) $\rightarrow \mathrm{HEX} \rightarrow \mathrm{NiGe}$

G5. Ni $(48.5 \% \mathrm{Ge}) \rightarrow \mathrm{HEX}+\mathrm{NiGe} \rightarrow \mathrm{NiGe}$

G6. Ni (49-50\%Ge) $\rightarrow$ NiGe 

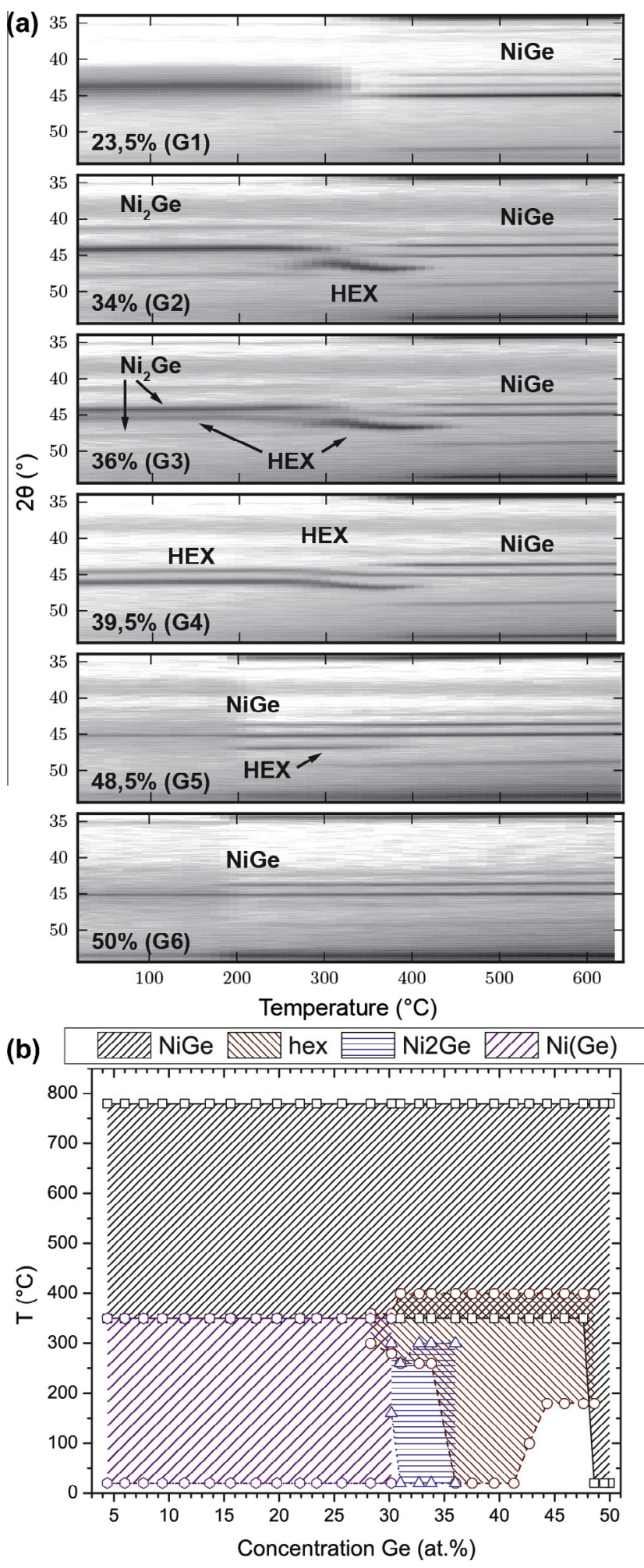

Fig. 3. (a) Selected in situ for annealing temperatures above XRD scans representing the different phase formation sequences observed on the $\mathrm{Ge}(100)$ samples during an anneal to $650^{\circ} \mathrm{C}$ at $3{ }^{\circ} \mathrm{C} / \mathrm{s}$. XRD intensity is plotted as a function of temperature $(x$-axis) and $2 \theta$ (y-axis) using a grayscale map with black representing the highest intensity. (b) Summary of phase formation for $\mathrm{Ni}(\mathrm{x} \% \mathrm{Ge})$ on $\mathrm{Ge}(100)$ samples. The shaded areas mark the regions in Ge concentration and temperature where the respective phase was detected with in situ XRD.

Again, the selected in situ XRD scans visible in Fig. 3(a) are representative for the different cases. Similar to what we have observed for the samples on $\mathrm{SiO}_{2}$, the HEX phase is forming below

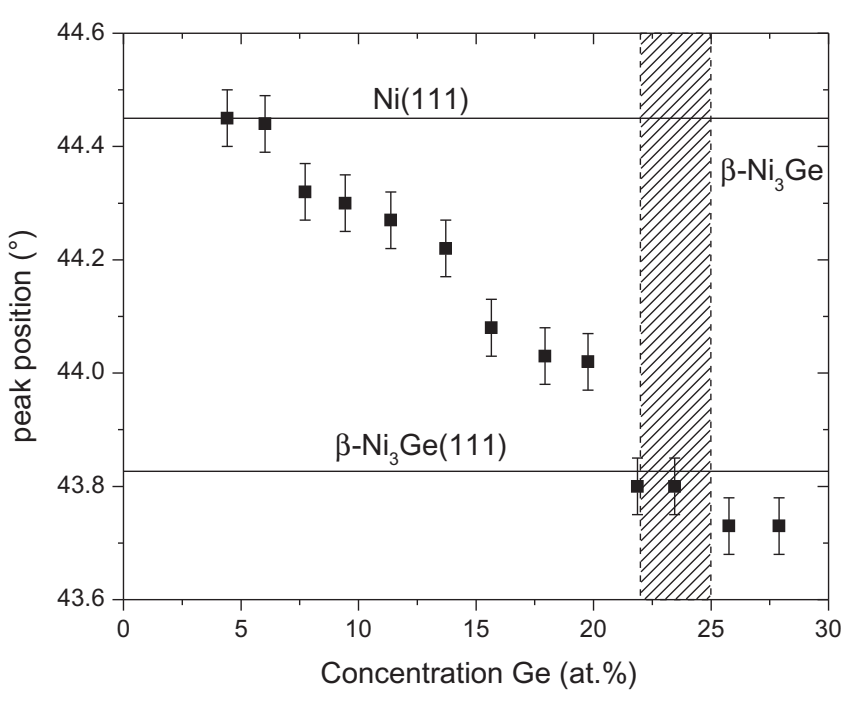

Fig. 4. Evolution of the measured peak position in the as-deposited Ni-Ge layers with Ge concentrations <28at.\% as a function of Germanium concentration. The JCPDS peak positions of $\mathrm{Ni}(111)$ and $\beta-\mathrm{Ni}_{3} \mathrm{Ge}(111)$ are indicated by the horizontal lines. The shaded area depicts the stability range of $\beta-\mathrm{Ni}_{3} \mathrm{Ge}$ on the binary phase diagram.

$300{ }^{\circ} \mathrm{C}$ for samples on $\mathrm{Ge}(100)$ with a Ge concentration between 36 and 48 at.\%. Also in this case, for samples with a Ge concentration between 36 and 42 at.\%Ge, the HEX phase is present in the asdeposited film (see Fig. 3)(b), again indicating the formation of this phase during the co-sputtering of $\mathrm{Ni}$ and $\mathrm{Ge}$ at room temperature.

\section{Discussion}

In recent years, detailed phase formation studies in the technologically relevant $\mathrm{Ni}$-silicon system have proven to be an important step in understanding the properties and behavior of Ni-silicide phases. For these planar metal thin film on silicon systems, it is generally accepted that phase formation initiates in a thin intermixed amorphous layer which forms at the metal-silicon interface during deposition. Consequently, we can assume that a similar process is true for metal-germanium systems. In this context, the amorphous $\mathrm{Ni}-\mathrm{Ge}$ layers studied in this work can be considered as simplified models for such an intermixed interface layer.

Based on the phase formation summary plots (Figs. 2(b) and 3(b)), some interesting observations can be made. First, the influence of the unlimited Ge supply from the substrate for the samples on $\mathrm{Ge}$ (100) is clearly visible. From Fig. 3(b) it can be seen that NiGe is always the stable end phase for annealing temperatures above $\sim 380^{\circ} \mathrm{C}$, which makes sense because an unlimited supply of Ge is expected to push the system towards the most Ge rich germanide phase. In contrast, for the samples on $\mathrm{SiO}_{2}$ the NiGe phase only forms when the Ge concentration in the intermixed films approaches 50 at.\%, which is the Ge content expected in stoichiometric NiGe.

For samples in the ranges 01 and $\mathrm{G} 1$, a single (broad) diffraction peak is visible around $2 \theta=44^{\circ}$ in the in situ XRD spectra from the as-deposited state on. This peak could be identified as either $\mathrm{Ni}$ (111) $\left(2 \theta=44.49^{\circ}\right)$ or $\beta-\mathrm{Ni}_{3} \mathrm{Ge}(111)\left(2 \theta=43.83^{\circ}\right)$ due to the closely related crystal structures of $\mathrm{Ni}$ and $\beta-\mathrm{Ni}_{3} \mathrm{Ge}$. In fact, $\beta-\mathrm{Ni}_{3} \mathrm{Ge}$ is essentially the Ni structure with site-ordered $\mathrm{Ni}$ atoms substituted with Ge, yielding a slightly larger lattice constant [5]. Fig. 4 shows this peak's measured position in the as-deposited layers as a function of Ge concentration and clearly reveals a gradual shift from $\mathrm{Ni}(111)$ to $\beta-\mathrm{Ni}_{3} \mathrm{Ge}$ (111) when approaching the stable composition range of the latter phase (according to the Ni-Ge phase 
diagram in Fig. 1, depicted by the shaded area in Fig. 4). The presence of this peak suggests that there is already some local structure in the co-deposited $\mathrm{Ni}-\mathrm{Ge}$ films that probably resembles the crystal structure of $\mathrm{Ni}$ and $\beta-\mathrm{Ni}_{3} \mathrm{Ge}$. The positional shift of the peak could then be caused by a solid-solution behavior of this intermixed phase, meaning that the addition of extra $\mathrm{Ge}$ in the codeposited film drives the local structure from $\mathrm{Ni}$ to $\beta-\mathrm{Ni}_{3} \mathrm{Ge}$ as the extra Ge gets systematically incorporated. Around $400^{\circ} \mathrm{C}$, for the samples on $\mathrm{SiO}_{2}$, a sudden improvement of the crystalline quality of this solid-solution phase is clearly evidenced by the intensity increase and narrowing of the peak around $44^{\circ}$ and the emergence of a second peak around $51^{\circ}$. For the samples on Ge (100), the intermixed phase transforms to $\mathrm{NiGe}$ at a similar temperature due to the unlimited supply of Ge from the substrate.

The crystallinity of the as-deposited films is even more pronounced for samples in the ranges $\mathrm{O} 2$ and G2. Here, multiple sharp diffraction peaks belonging to $\mathrm{Ni}_{2} \mathrm{Ge}$ were found to be present in the co-deposited films, suggesting the presence of fully crystalline $\mathrm{Ni}_{2} \mathrm{Ge}$ grains in these films (see Figs. 2(a) and 3(a)).

The most interesting composition region comprises the ranges O3 through 06 for the samples on oxide and G3 through G5 for the samples on $\mathrm{Ge}(100)$, i.e. the compositional range between 36 and 48 at.\%Ge. In situ XRD revealed two clear diffraction peaks around $45^{\circ}$ and $46.5^{\circ}$ for temperatures below $300^{\circ} \mathrm{C}$. For Ge concentrations in the range $[36,42]$ at.\% these peaks are present from the as-deposited state on, again indicating the existence of crystalline grains in these co-deposited films. According to the Ni-Ge phase diagram (Fig. 1), four phases are expected to be stable below $300{ }^{\circ} \mathrm{C}$ and thus could explain the presence of these diffraction peaks: $\beta-\mathrm{Ni}_{3} \mathrm{Ge}, \mathrm{Ni}_{2} \mathrm{Ge}, \mathrm{NiGe}$ and the low-temperature $\epsilon^{\prime}-\mathrm{Ni}_{5} \mathrm{Ge}_{3}$ phase. While neither of the two peaks can be attributed to one of the first three phases, they could be explained by superpositions of the $\epsilon^{\prime}-\mathrm{Ni}_{5} \mathrm{Ge}_{3}(203) /(313)$ and (602)/(331) peaks. Nonetheless, according to the JCPDS data, a lot more diffraction peaks are expected for this $\epsilon^{\prime}-\mathrm{Ni}_{5} \mathrm{Ge}_{3}$ phase in the measured $2 \theta$ window, especially since we do not expect any texture effects on the $\mathrm{SiO}_{2}$ substrates. Unexpectedly, further investigation of this in situ XRD data below $300{ }^{\circ} \mathrm{C}$ revealed a perfect agreement with the diffraction pattern that is expected for the high-temperature, hexagonal $\epsilon-\mathrm{Ni}_{5} \mathrm{Ge}_{3}$ and $\mathrm{Ni}_{3} \mathrm{Ge}_{2}$ phases (which we treat together as a single 'HEX' phase as mentioned above), i.e. all expected diffraction peaks were present and no extra peaks were found. This was confirmed with in situ XRD scans taken in a complementary $2 \theta$ window (data not shown here). Since this HEX phase is not expected to be stable below $300{ }^{\circ} \mathrm{C}$ according to the Ni-Ge phase diagram (Fig. 1), these findings suggest the presence of metastable HEX grains in these films below $300^{\circ} \mathrm{C}$.

To confirm the presence of these metastable HEX grains, we performed a high-resolution TEM (HR-TEM) measurement combined with electron diffraction on a sample in the range G4 that was quenched at $120^{\circ} \mathrm{C}$. The results are shown in Fig. 5. The HRTEM image (Fig. 5(a)) shows a crystalline Ni-Ge film on top of the $\mathrm{Ge}(100)$ substrate. An electron diffraction pattern collected in the indicated area is shown in Fig. 5(b), confirming a hexagonal crystal structure. Fig. 5(c) shows a simulated diffraction pattern of this HEX phase using the lattice parameters known from literature (spacegroup $\mathrm{P} 6_{3} / \mathrm{mmc} ; \quad a=b \approx 3.9 \AA ; c=5.078 \AA ; \alpha=\beta=90^{\circ}$; $\gamma=120^{\circ}$ ) [3,13]. A good agreement between the measured and simulated patterns can be observed, reinforcing the suggested presence of metastable HEX grains in the co-deposited films. Additional HR-TEM results (not shown here) confirm the presence of this phase throughout virtually the whole Ni-Ge layer. From this observation, it can be assumed that the aforementioned two diffraction peaks around $45^{\circ}$ and $46.5^{\circ}$ that were visible in in situ XRD scans of samples in the range $[36,48]$ at.\%Ge are caused by HEX (102) and (110) diffractions respectively. The in situ XRD

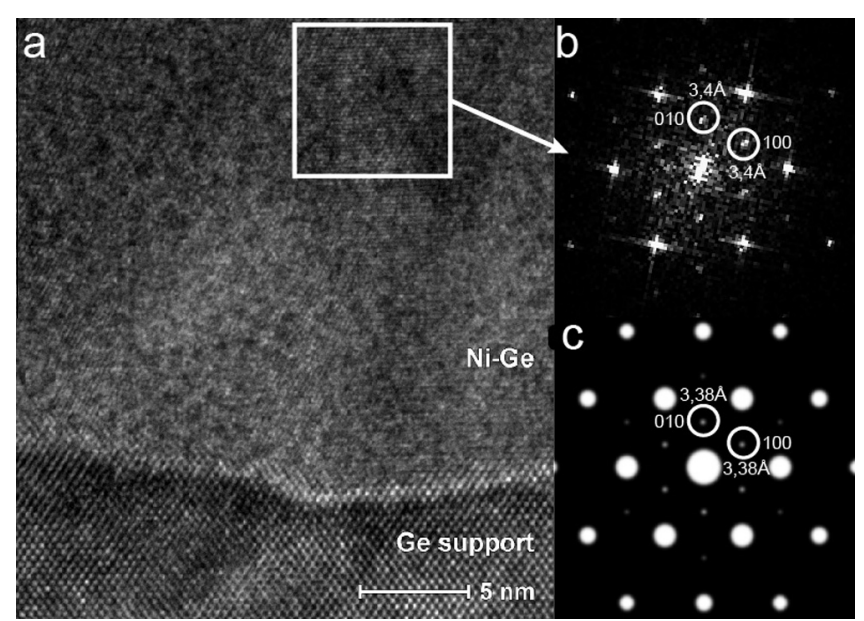

Fig. 5. (a) HR-TEM image of a $\mathrm{Ni}(38 \% \mathrm{Ge})$ film on $\mathrm{Ge}(100)$ quenched at $120^{\circ} \mathrm{C}$. (b) Electron diffraction pattern recorded within the indicated area. A similar diffraction pattern could be observed virtually everywhere throughout the film. (c) Simulated diffraction pattern for the HEX crystal structure as known from literature (spacegroup $\mathrm{P}_{3} / \mathrm{mmc} ; a \approx 3.9 \AA ; c \approx 5.078 \AA$ ).

results thus suggest the metastable occurrence of this HEX phase in the wide compositional range between 36 and 48 at.\%Ge. Between 36 and 42 at.\%Ge, the crystallization of this phase occurs already during deposition.

Next, we focus on the influence of Ge content on the HEX crystal structure. In the metastable region discussed above, a systematic shift of the (102) and (110) peaks towards higher diffraction angles over a range of $\sim 2^{\circ}$ could be observed in the in situ XRD measurements, both for the samples on $\mathrm{SiO}_{2}$ as for those on $\mathrm{Ge}(100)$. From the positions of these two diffraction peaks (as determined from the in situ XRD scans at $200^{\circ} \mathrm{C}$ ), the lattice parameters $a$ and $c$ of the HEX phase can be calculated using Bragg's law and the relation between $d$-spacing and lattice constants for a hexagonal crystal structure. The influence of the Ge content in the co-deposited $\mathrm{Ni}-\mathrm{Ge}$ film on the lattice of the metastable HEX phase is presented in Fig. 6, which shows a systematic contraction of the lattice with increasing Ge content. A similar behavior has been observed previously by Ellner et al. in the bulk system [3] and by Jensen et al. in multilayer Ni-Ge films [8]. Since the structure of this HEX phase

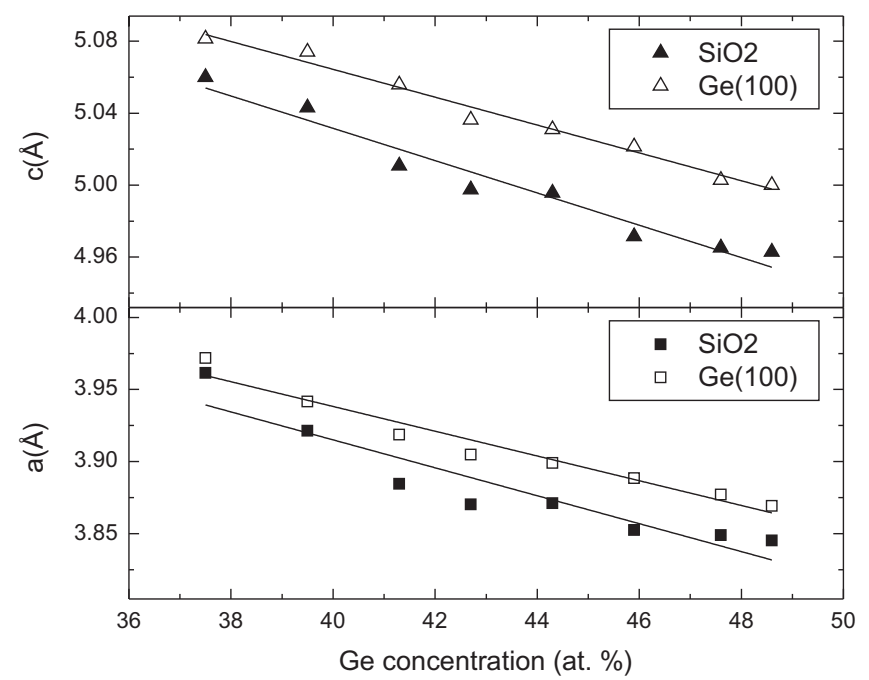

Fig. 6. Influence of the Ge content in the co-deposited film on the lattice parameters a and c of the metastable HEX crystal structure, both on $\mathrm{SiO}_{2}$ and $\mathrm{Ge}$ (100). The parameters were calculated from the HEX (102) and (110) peak positions as extracted from the in situ XRD scans at $200{ }^{\circ} \mathrm{C}$. 
can be regarded as intermediate between the two prototype structures of $\mathrm{Ni}_{2} \mathrm{In}$ and $\mathrm{NiAs}$, the monotonic decrease of the lattice parameters can be explained by a systematic incorporation of extra vacancies on the Ni sites as more Ge is added to the mixture $[13,8]$. The observation of this metastable HEX phase and its behavior as a function of Ge content is very similar to what has been observed recently for the $\theta$-phase in the $\mathrm{Ni}-\mathrm{Si}$ system [11].

Finally, the behavior of this HEX phase with increasing Ge content also explains the continuous shift of the HEX (102) and (110) diffraction peaks between 300 and $400{ }^{\circ} \mathrm{C}$ that was observed for various samples on Ge (100) (see Fig. 3(a)). First, the HEX phase nucleates with a certain composition out of the co-deposited film as mentioned above. Then, when the thermal budget supplied to the sample is high enough to allow for a reaction between the substrate and the film (in this case around $300{ }^{\circ} \mathrm{C}$ ), additional Ge from the substrate gets incorporated into the HEX phase, inducing a lattice contraction and a corresponding peak shift towards higher $2 \theta$ diffraction angles. Additional TEM measurements on a sample with 38 at.\%Ge quenched after the peak shift (not shown here) confirmed the presence of HEX grains with similar electron diffraction patterns than those observed for HEX grains at $120^{\circ} \mathrm{C}$. This confirms our assumption that this peak shift is not due to a phase transformation but to a monotonic compositional change of this HEX phase as explained above.

\section{Conclusions}

A detailed study of phase formation in co-deposited $\mathrm{Ni}-\mathrm{Ge}$ thin films with $\mathrm{Ge}$ concentrations ranging from 0 to 50 at.\% on both $\mathrm{SiO}_{2}$ and Ge (100) substrates was carried out using in situ XRD and TEM. A combinatorial sputter deposition technique allowed for the deposition of all investigated samples in a single run. Crystalline phases were found to nucleate already during co-deposition of the films at room temperature for almost the full composition range under investigation. Between 36 and 48 at.\%Ge, a metastable high-temperature hexagonal germanide $\left(\epsilon-\mathrm{Ni}_{5} \mathrm{Ge}_{3}\right.$ or $\mathrm{Ni}_{3} \mathrm{Ge}_{2}$ in the binary phase diagram, but treated as a single 'HEX' phase in this work) was found to be present below its minimum stability temperature of $300^{\circ} \mathrm{C}$, adapting its crystal structure to the available amount of Ge. Between 36 and 42 at.\%Ge, this HEX phase was present already in the as-deposited state.

\section{Acknowledgments}

The author acknowledges the 'Fonds voor Wetenschappelijk Onderzoek Vlaanderen' (FWO) for financial support through a scholarship and project Nr. G076112N.

\section{References}

[1] R. Pillarisetty, Nature 479 (2011) 324-328.

[2] S. Gaudet, C. Detavernier, A.J. Kellock, P. Desjardins, C. Lavoie, Journal of Vacuum Science \& Technology A 24 (2006) 474

[3] M. Ellner, T. Gödecke, K. Schubert, Journal of the Less Common Metals 24 (1971) 23-40.

[4] A. Dayer, P. Feschotte, Journal of the Less Common Metals 72 (1980) 51-70.

[5] A. Nash, P. Nash, Bulletin of Alloy Phase Diagrams 8 (1987) 255-264.

[6] S. Gaudet, C. Detavernier, C. Lavoie, P. Desjardins, Journal of Applied Physics 100 (2006) 034306

[7] F. Nemouchi, D. Mangelinck, C. Bergman, G. Clugnet, P. Gas, J.L. Lábár, Applied Physics Letters 89 (2006) 131920.

[8] J. Jensen, S. Ly, D. Johnson, Chemistry of Materials 15 (2003) 4200-4204.

[9] K. De Keyser, C. Van Bockstael, C. Detavernier, R.L. Van Meirhaeghe, J. JordanSweet, C. Lavoie, Electrochemical and Solid State Letters 11 (2008) H266.

[10] C. Van Bockstael, K. De Keyser, R.L. Van Meirhaeghe, C. Detavernier, J.L. JordanSweet, C. Lavoie, Applied Physics Letters 94 (2009) 033504.

[11] C. Van Bockstael, C. Detavernier, R.L. Van Meirhaeghe, J.L. Jordan-Sweet, C. Lavoie, Journal of Applied Physics 106 (2009).

[12] S. Gaudet, P. Desjardins, C. Lavoie, Journal of Applied Physics 110 (2011) 113524.

[13] A.-K. Larsson, R. Withers, Journal of Alloys and Compounds 264 (1998) 125132. 